\title{
Testing the Gaussianity and Statistical Isotropy of the Universe
}

\author{
Dragan Huterer, ${ }^{1}$ Eiichiro Komatsu, ${ }^{2}$ and Sarah Shandera ${ }^{3}$ \\ ${ }^{1}$ Department of Physics, University of Michigan, 450 Church Street, Ann Arbor, MI 48109-1040, USA \\ ${ }^{2}$ Texas Cosmology Center and Department of Astronomy, The University of Texas at Austin, Austin, TX 78712, USA \\ ${ }^{3}$ Perimeter Institute for Theoretical Physics, 31 Caroline Street North, Waterloo, ON, Canada N2L 2Y5 \\ Correspondence should be addressed to Dragan Huterer, huterer@umich.edu
}

Received 31 December 2010; Accepted 31 December 2010

Copyright (C) 2010 Dragan Huterer et al. This is an open access article distributed under the Creative Commons Attribution License, which permits unrestricted use, distribution, and reproduction in any medium, provided the original work is properly cited.

Background and Motivation. The last few years have seen a surge in excitement about measurements of statistics of the primordial fluctuations beyond the power spectrum. New ideas for precision tests of Gaussianity and statistical isotropy in the data are developing simultaneously with proposals for a wide range of new theoretical possibilities. From both the observations and theory, it has become clear that there is a huge discovery potential from upcoming measurements.

The twin principles of statistical isotropy and homogeneity are a crucial ingredient in obtaining most important results in modern cosmology. For example, with these assumptions temperature and density fluctuations in different directions on the sky can be averaged out, leading to accurate constraints on cosmological parameters that we have today. Nevertheless, there is no fundamental reason why these must be obeyed by our universe. Statistical isotropy and homogeneity are starting to be sharply tested using the cosmic microwave background (CMB) and large-scale structure data. Recently, there has been particular activity in these areas, given Wilkinson Microwave Anisotropy Probe remarkable maps, combined with claims of large-angle "anomalies" indicating departures from statistical isotropy as predicted by standard inflationary models.

The statement that primordial curvature fluctuations are nearly Gaussian on scales measured by the CMB is remarkably precise but does not reveal much about their source. Current constraints on the amplitude of the threepoint correlation function of fluctuations are nearly four orders of magnitude above predictions from single field slowroll inflation models and at least an order of magnitude above what is expected just from nonlinearities that develop after the primordial spectrum is laid down. There are a wide spectrum of interesting models that can be ruled out by tightening this constraint; conversely, a detection of nonGaussianity would rule out single field slow-roll inflation. While current observations of the CMB fluctuations provide reasonably strong evidence for a primordial source of fluctuations from inflation, only measurements of higherorder statistics can truly shed light on the physics of inflation.

Departures from statistical isotropy and Gaussianity involve a rich set of observable quantities, with diverse signatures that can be measured in the $\mathrm{CMB}$ or in largescale structure using sophisticated statistical methods. These signatures, which carry information about physical processes on cosmological scales, have power to reveal detailed properties of the physics responsible for generating the primordial fluctuations. Even qualitative observational features can identify key properties of the fields involved (e.g., how many fields and which couplings were most relevant) or, alternatively, shed light on the systematic errors in the data. However, because there are so many possibilities from both theory and observation and because many calculations are very technical involving methods such as higher-order perturbation theory, the literature can be daunting.

In this special issue, we have collected articles that summarize the theoretical predictions for departures from Gaussianity or statistical isotropy from a variety of potential sources, together with the observational approaches to test these properties using the $\mathrm{CMB}$ or large-scale structure. We hope this collection provides an accessible entry point to these topics as they currently stand, indicating what direction future developments may take place and demonstrating why these questions are so compelling.

Dragan Huterer Eiichiro Komatsu Sarah Shandera 

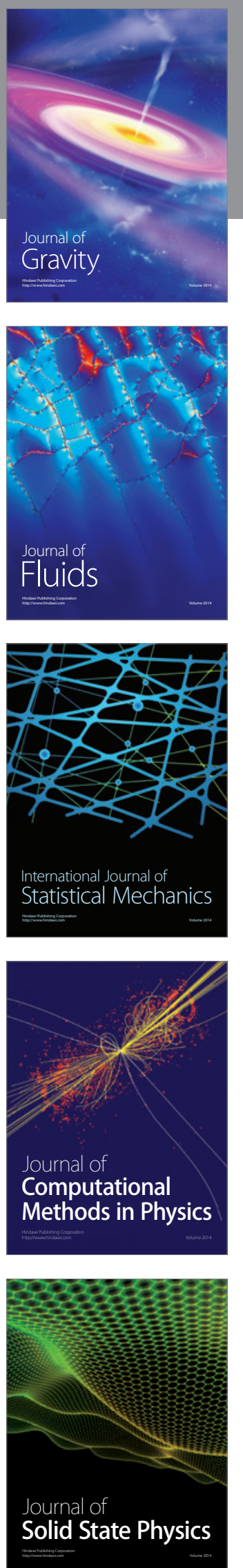

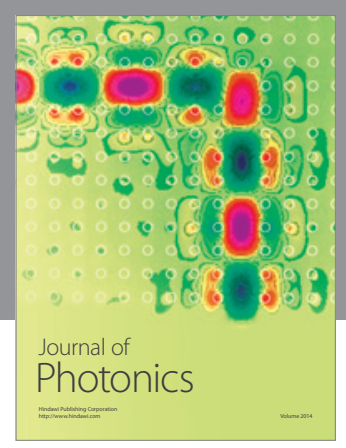

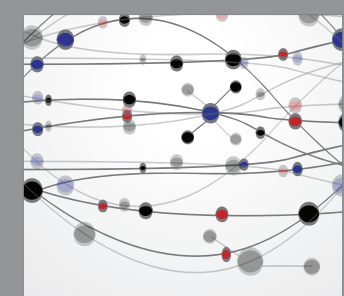

The Scientific World Journal
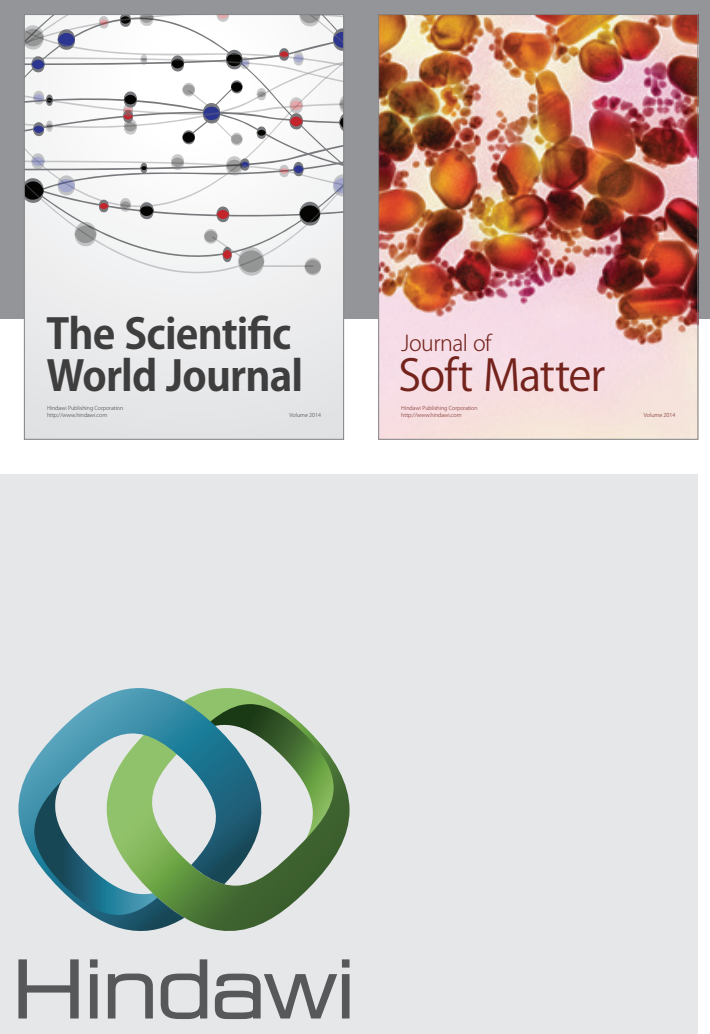

Submit your manuscripts at

http://www.hindawi.com
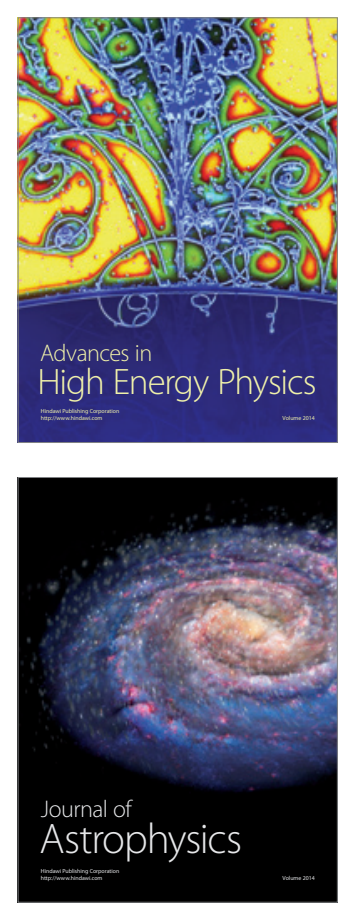
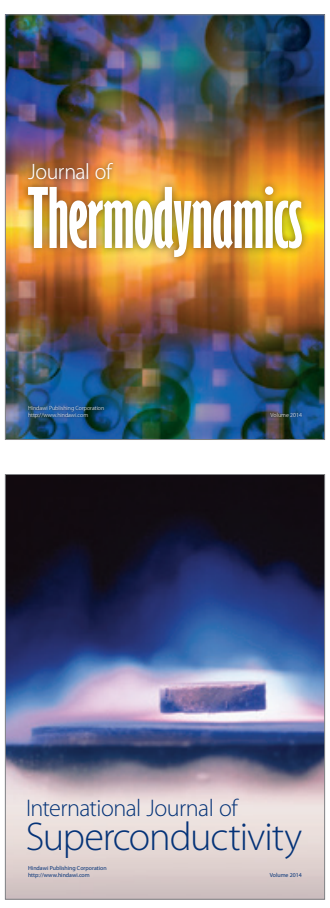
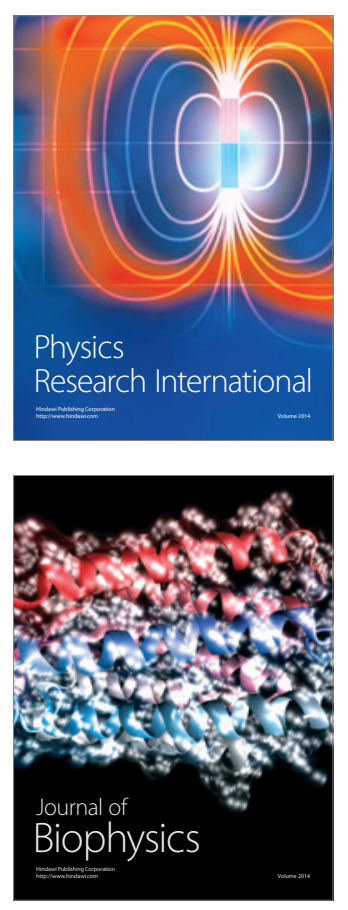
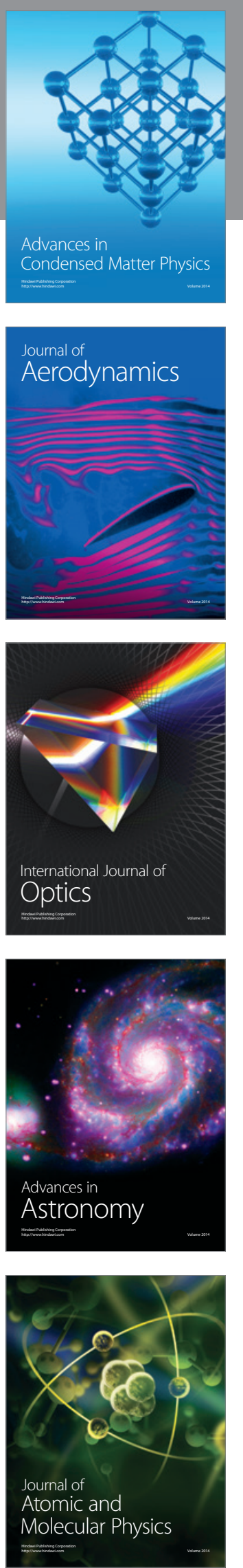